\title{
Chemical Specific Imaging and Spectroscopy of Interfaces and Dynamic Surface Processes with Synchrotron-Based X-ray Microscopy *
}

\author{
Maya Kiskinova ${ }^{\dagger}$ \\ Sincrotrone Trieste, Area Science Park, Basovizza, 34012-Trieste, Italy \\ (Received 5 October 2003; Accepted 24 October 2003; Published 19 January 2004)
}

\begin{abstract}
The paper is a brief overview of the potential of the photoemission microscopy methods emphasizing on some recent achievements of chemical imaging and spectromicroscopy in identification of sub-micron area chemical phases developed as a result of surface reactions, propagating reaction fronts and mass transport due to thermal and/or electromigration. [DOI: 10.1380/ejssnt.2004.1]
\end{abstract}

Keywords: Synchrotron radiation photoelectron spectromicroscopy; Surface reactions; Surface diffusion

\section{INTRODUCTORY REMARKS ON X-RAY MICROSCOPY APPROACHES}

It has long ago been recognized that lateral inhomogeneity in the composition and/or morphology can exert a dramatic effect on the interface properties. The demand to have access to the fluctuations in the chemical composition, electronic and magnetic structure of surfaces and interfaces at submicron and mesoscopic length scales has been met to a great extend by the construction of different types of $\mathrm{x}$-ray photoemission microscopes [1]. The contrast mechanisms in these instruments are based on photon absorption and photon-induced electron emission. The element specific, chemical, electronic and magnetic information is obtained using the conventional spectroscopy methods, namely X-ray absorption spectroscopy (XAS) and X-ray photoelectron spectroscopy (XPS), adding spatial resolution intermediate between light microscopy and electron microscopy. The $\mathrm{x}$-ray spectromicroscopy techniques have undergone very fast progress with the construction of low emittance synchrotron radiation machines, which provide energy tunable photon beams with very high brightness and various polarization. This review article will illustrate the potential of x-ray microscopes using X-ray Photoelectron Spectroscopy (XPS) to obtain chemically-specific information about processes occurring at solid surfaces and interfaces.

XPS is a very surface sensitive technique and is very appropriate for investigations of interfacial phenomena: the sample probing depth is determined by the kinetic energy of the photo-emitted electrons and using soft $\mathrm{x}$ rays usually does not exceed $100 \AA$. In the modern xray photoemission microscopes high spatial resolution is achieved using two different approaches, magnifying the image of the irradiated surface area or demagnifying the incident photon beam. In the instruments using the first approach (called X-ray PhotoEmission Electron Microscopes - XPEEM) suitable electrostatic or magnetostatic electron optical imaging system for magnification and projection of the emitted electron are utilized. In the instruments using the second approach the photon beam is demagnified to submicrometer dimensions using suitable

\footnotetext{
* This paper was presented at The 4th International Symposium on Atomic Level Characterizations for New Materials and Devices (ALC '03), Kauai, Hawaii, USA, 5-10 October, 2003.

${ }^{\dagger}$ Corresponding author: maya.kiskinova@elettra.trieste.it
}

photon optics. They are called Scanning PhotoEmission Microscopes (SPEM), because the images are formed by scanning the sample with respect to the focused beam.

At present three beamlines of the synchrotron light facility Elettra host photoemission microscopes. The imaging microscope, XPEEM, purchased by ELMITEC, has an energy filter which adds XPS to the X-ray Absorption Spectroscopy (XAS) used in the standard PEEM instruments, which monitor the total emitted electron signal while varying the energy of the incident photon beam [2]. In PEEM the lateral resolution and spectral resolution are 'inversely' correlated because of the instrument transmission, so that the analyzer energy resolution should be relaxed when very high lateral resolution is required. An important advantage of XPEEM, in addition to the high lateral resolution and direct imaging abilities, is that its design allows implementing an electron gun to add complementary methods for structural analysis, namely low energy electron microscopy (LEEM) and low energy electron diffraction (LEED). The scanning microscopes use refractive near-normal-incidence optics, Schwarzshield objective (SPEM-1) and diffractive optics, high resolution Fresnel zone plate (ZP) lenses (SPEM-2) to obtain a microprobe. The two scanning instruments are complementary; SPEM-1 works well at photon energies less than $\sim 100 \mathrm{eV}$ and is very well suited for valence band mapping, whereas SPEM-2 works exclusively with photon energies above $300 \mathrm{eV}$ achieving excellency in core level spectroscopy measurements. In the present report I will show only results obtained with SPEM-2. Lateral and spectral resolution of SPEM instruments are independent, the former being determined only by the focusing optics is the same in imaging and spectroscopy mode. In SPEM instruments it turned out to be very helpful the implementation of a multichannel detector, because it reduces substantially the time necessary for getting chemically specific and topographic information. In multichannel detection, the number of images collected simultaneously is equal to the number of the channels, where each channel corresponds to a specific kinetic energy, defined by the selected energy window. The multi-channel imaging allows not only mapping of different chemical states and the background with a single scan of the sample but also reconstruction of the spectrum corresponding to the energy window, so-called spectro-imaging. The performance of the multichannel detector can be improved by increasing the number of parallel counting channels. Figure 1 illustrates the most recent achievements in spectroimaging using 48-channel detector. The example is an 


\section{Spectro-imaging}

(a)

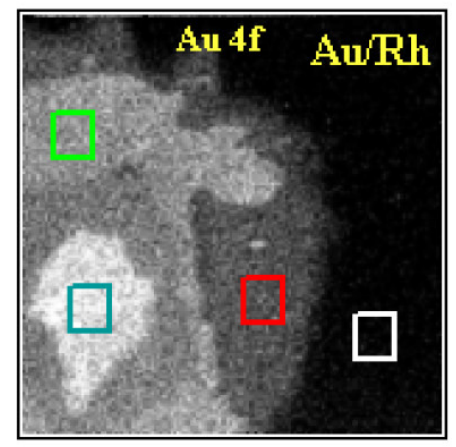

(b)

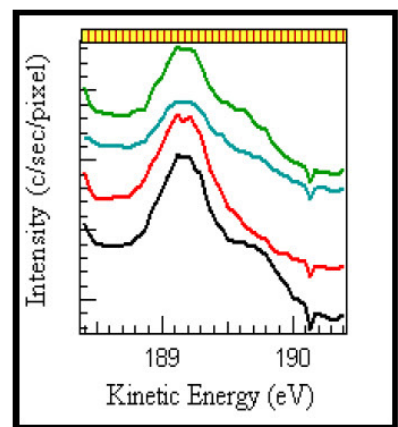

spectroscopy

(c)

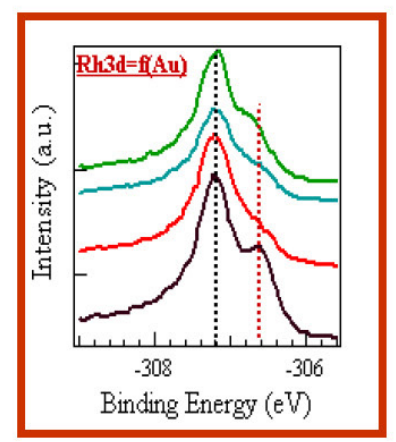

FIG. 1: (a) Au 4f map $\left(64 \times 64 \mu \mathrm{m}^{2}\right)$ illustrating areas with different Au coverage on a Rh(110) surface. (b) Reconstructed spectra from the corresponding to (a) $\mathrm{Rh} 3 \mathrm{~d}_{5 / 2}$ image from the areas indicated with colored squares in (a). (c) $\mathrm{Rh} 3 \mathrm{~d}_{5 / 2}$ spectra measured in spectroscopy mode from a microspot in the center of the squares.
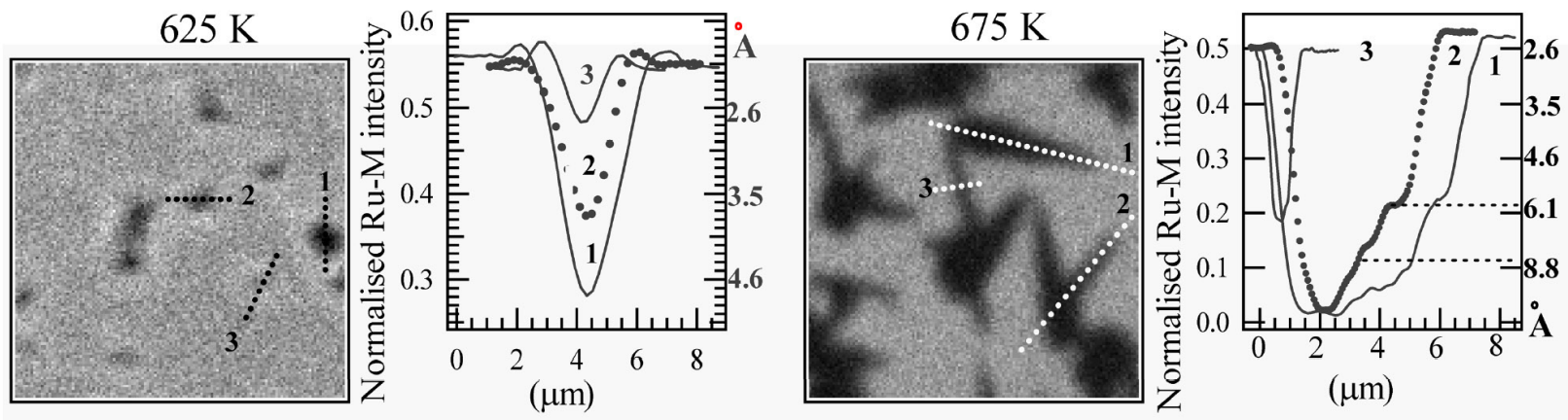

FIG. 2: Ru-M chemical maps $\left(25.6 \times 25.6 \mu \mathrm{m}^{2}\right.$ and $\left.12.8 \times 12.8 \mu \mathrm{m}^{2}\right)$ and plots of selected intensity profiles taken along the lines indicated with 1, 2 and 3 in the images, normalized with respect to the intensity of the $\mathrm{Ru} 3 \mathrm{~d}_{5 / 3}$ emission of clean $\mathrm{Ru}(0001)$ before oxidation: The right y-scale of the profile plots indicates the calculated oxide thickness. The O-depleted zones around the oxide islands formed at $625 \mathrm{~K}$ are evidenced by the two small maxima on both sides of the Rh-M profiles.

$\mathrm{Au} / \mathrm{Rh}(110)$ interface, where the Rh $3 \mathrm{~d}$ spectra undergo quite significant lineshape changes as a function of the local Au coverage. The panel (a) shows the Au $4 \mathrm{f}$ map, where the contrast level reflects the lateral variations of the $\mathrm{Au}$ coverage. From the $\mathrm{Rh} 3 \mathrm{~d}_{5 / 2}$ map of the same area (not shown) we reconstructed the $\mathrm{Rh} 3 \mathrm{~d}_{5 / 2}$ spectra from the indicated areas, which are shown in panel (b). Panel (c) shows the Rh $3 \mathrm{~d}_{5 / 2}$ spectra measured in a conventional spectroscopy mode from a spot within the indicated areas. The striking similarity in energy resolution achieved using spectro-imaging is evident. It should be noted that increasing the number of parallel counting channels the pass energy of the analyzer can be increased accepting a much wider energy range of the emitted photoelectrons, while maintaining the same energy resolution on each channel.

Table 1 summarizes the major characteristic parameters of the imaging and scanning instruments, which manifest the advantages and disadvantages of each of these approaches.

There are certainly many problems that cannot be solved with XPEEM or SPEM alone, so that combining the presently available instrumentation for spectromicroscopy is the best research approach.

\section{SOME REPRESENTATIVE EXAMPLES ILLUSTRATING THE POTENTIAL OF CHEMICAL IMAGING AND \\ SPECTROMICROSCOPY USING SPEM AND/OR XPEEM INSTRUMENTS}

The information that one wants to obtain using XPS imaging and spectromicroscopy can be summarized as follows. Most often the focus is on correlation between the contrast and the local elemental composition or chemical state of the element of interest. In this case the emission from a specific electronic level of the element is mapped, the so-called core level energy shifts and changes in the valence spectra being fingerprints of the actual chemical state and electronic structure. The few selected research topics focused on surface reactions and mass transport phenomena will illustrate the variety of useful information obtained by examining the chemical composition of complex interfaces with submicrometer resolution.

\section{A. Initial oxidation stage of a $\mathrm{Ru}(0001)$ surface}

The first example tackles the microscopic structure of transition metal catalysts under reaction conditions. The 
TABLE I: Major characteristic parameters of the imaging and scanning instruments.

\begin{tabular}{|c|c|c|c|}
\hline & & SPEM & XPEEM \\
\hline \multirow[t]{2}{*}{ Imaging } & Lateral resolution & $\begin{array}{l}\sim 100 \mathrm{~nm} \text { at present, } 40 \sim 50 \mathrm{~nm} \text { feasible } \\
\text { with better zone plate optics. }\end{array}$ & $\begin{array}{l}10 \sim 20 \mathrm{~nm} \text { at present, } 2 \mathrm{~nm} \text { feasible adding } \\
\text { aberration correction systems. }\end{array}$ \\
\hline & Acquisition rate & $\begin{array}{l}\text { Usually in a minute range for moderate } \\
\text { pixel size, the same in single and multichan- } \\
\text { nel imaging. }\end{array}$ & $\begin{array}{l}\text { Usually in a sec range. Subsequent record- } \\
\text { ing of images for chemical imaging slows the } \\
\text { rate. }\end{array}$ \\
\hline \multirow[t]{2}{*}{ Spectroscopy } & $\begin{array}{r}\text { Micro-area } \\
\text { Energy resolution }\end{array}$ & $\begin{array}{l}\sim 0.01 \mu \mathrm{m}^{2} \text { at present, } \sim 0.002 \mu \mathrm{m}^{2} \text { feasible. } \\
\text { can become even less than } 10 \mathrm{meV} \text { using } \\
\text { highly monochromatic photons and good } \\
\text { analyser. }\end{array}$ & $\begin{array}{l}\sim 1 \mu \mathrm{m}^{2} \text {, submicron feasible. } \\
\sim 400 \mathrm{meV} \text { at present (depends on the ana- } \\
\text { lyzer and the photon flux density). }\end{array}$ \\
\hline & Acquisition rate & $\begin{array}{l}\text { Usually in a min range for high-energy res- } \\
\text { olution mode. Snapshot multichannel de- } \\
\text { tection mode can work in a sec range. }\end{array}$ & $\begin{array}{l}\text { From sec to minute range, depending on the } \\
\text { working conditions (low flux density but } \\
\text { large probed area). }\end{array}$ \\
\hline
\end{tabular}

question that has barely been addressed is what determines the catalyst activity and selectivity in oxidation reactions - the properties of the bare metal surface or the metastable, e.g. 'oxide' microphases which can be formed in the presence of oxidizing reagents. $\mathrm{Ru}$, along with $\mathrm{Pt}$ and $\mathrm{Rh}$, is among the catalysts that have attracted the interest of the surface scientists in an attempt to verify the mechanism of catalytic $\mathrm{CO}$ oxidation and NO reduction in the automobile exhaust converters. The first important step to understanding the mechanism of catalytic action is verification of microscopic morphology of the Ru catalyst surface under reaction conditions. The pioneering studies combining structural imaging techniques, such as STM, I-V LEED and PEEM, with XPS, suggested that the 'active' Ru surface is O-rich and consists of different phases with microscopic dimensions including non-stoichiometric oxide phases and dissolved oxygen [3, 4]. However, neither of the used methods has provided sufficient qualitative and quantitative data confirming the assignment of the phases coexisting on the O-rich $\mathrm{Ru}$ surface and their catalytic activity. Recent SPEM study on development, spatial distribution and actual chemical state of the phases present on the so-called 'O-rich' $\mathrm{Ru}$ surface, has identified for the first time the local composition of the phases coexisting in the initial stages of the $\mathrm{Ru}(0001)$ oxidation, a quantitatively new information which is a solid base to verifying their actual role in $\mathrm{CO}$ oxidation [5]. For obtaining contrast in the chemical imaging the chemical shift undergone by the $\mathrm{Ru} 3 \mathrm{~d}$ core levels of the Ru atoms, which are bonded to oxygen, was used. As a result the $\mathrm{Ru} 3 \mathrm{~d}$ spectra contain a contribution of the photoelectrons emitted from the $\mathrm{Ru}$ atoms which are in a metallic state (below the formed oxide), $\mathrm{Ru}-\mathrm{M}$, and $\mathrm{Ru}$ atoms bonded to oxygen. Since the oxygen coordination number determines the core level energy shift this has allowed us to distinguish between stoichiometric $\mathrm{RuO}_{2}$, intermediate $\mathrm{Ru}$ sub-oxides and chemisorption phases. Figure 2 shows two $\mathrm{Ru} 3 \mathrm{~d}_{5 / 2}$ maps measured after exposure of the $\mathrm{Ru}(0001)$ sample to $10^{5} \mathrm{~L}$ of oxygen at $625 \mathrm{~K}$ and 675 $\mathrm{K}$, respectively, which 'evisualize' the spatial anisotropy of the initial stages of oxide growth and the dependence of the morphology on the oxidation temperature. The images in Fig. 2 are obtained measuring only the $\mathrm{Ru}$ $3 \mathrm{~d}$ signal from the metallic substrate $(\mathrm{Ru}-\mathrm{M})$, which decreases exponentially with increasing the thickness of the O-containing layer, so that the intensity profiles taken along the 'oxide patches' reflect the spatial variation in their thickness. This has allowed us to quantify the thickness of the $\mathrm{Ru}$ oxide formed under varying oxidation conditions normalizing the intensity profiles against the $\mathrm{Ru}$ $3 \mathrm{~d}$ emission measured for the clean $\mathrm{Ru}$ surface before oxidation. The elucidated thickness is shown in the right $y$-scale of the line profile graphs. They indicate that the 'oxide-like' phase formed at $625 \mathrm{~K}$ is at most two layers, whereas its thickness exceeds 3 layers in phase formed at $675 \mathrm{~K}$. An interesting feature of the $675 \mathrm{~K}$ growth is that after nucleation the oxide growth expands in a preferred crystallographic direction. The profiles taken across the protruding oxide features of the ' $675 \mathrm{~K}$ ' phase reveal the expected decrease of the thickness of the film in the oxide growth direction (from left to right). However, closer inspection distinguishes breaks in the slope on the side of the growth direction between 5 and $6 \AA$, and around 8 $\AA$, corresponding to $\sim 2$ and 3 oxide layers, respectively. This apparently reflects a layer-by-layer growth of $\mathrm{RuO}_{2}$. Note also that for a thickness less than two layers the initial slopes of all profiles are similar and do not show directional anisotropy. Another notable feature of this earliest oxidation stage is that the oxide nucleus is decorated by $\mathrm{O}$-depleted areas, which we tentatively ascribed to the finite mobility of the $\mathrm{O}$ atoms or $\mathrm{RuO}_{x}$ clusters.

\section{B. Mass transport of $\mathrm{Ga}$ on $\mathrm{GaN}$ induced by applied electric field}

The morphology of the surface or the interface can be considerably affected through a diffusion bias created by the electric field application, a phenomenon known as surface electromigration (SE) [5]. Coupled to a complex surface dynamics it can lead to qualitatively different scenarios of surface behavior. In the case of adsorbates and thin films the direction with respect to the electric field is specific to the individual atoms, the migration rate being coverage dependent and, as demonstrated by the present case, the mobility of the first interfacial layer and top layers can be different. A principle difficulty in quantitative description of SE is that the forced directional motion due to electric field is accompanied by the multi-directional thermal diffusion and the latter may dominate at high temperatures. Another obstacle is that the straightforward application of atomic scale models of diffusion for 
(a)

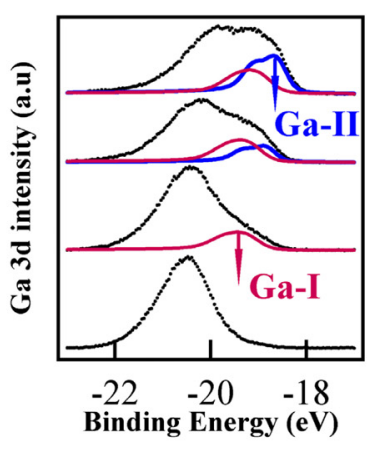

(b)

Initial state
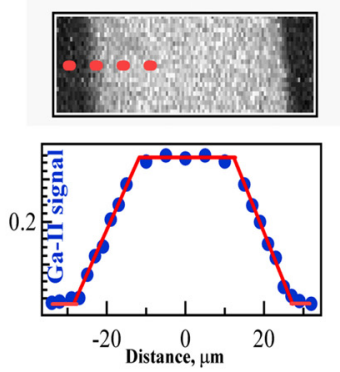

(c)

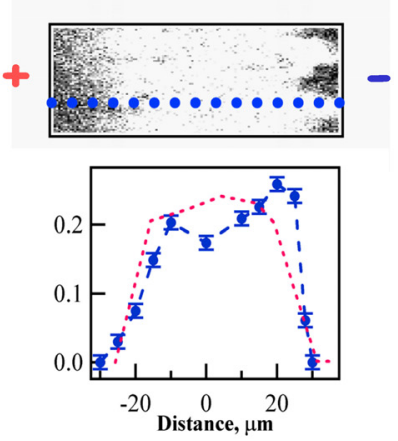

FIG. 3: (a) Deconvoluted Ga 3d spectra taken across the Ga patch, shown by the Ga 3d map, where the different energy position of interfacial Ga, Ga-I, and the second layer Ga, Ga-M are clearly seen. The concentration profile of Ga-I + Ga-M, has the expected trapezoid shape, determined by the distance between the sample and the mask used for the patch deposition. (b) Ga-M map and the corresponding Ga-M concentration profile developed as a result of Ga-I surface electromigration occurring only on top of the interfacial Ga-I layer. (c) Ga-M map and the corresponding Ga-M concentration profile developed as a result of mixed electromigration and thermal diffusion, where the expansion of the Ga patch is clearly seen. The red dotted line shows the profile developed in the absence of electric field.

describing the transport phenomena occurring at a larger meso- and microscopic scales is impeded by the imperfections, defects and irregularities present on the substrate surface and within the films deposited on top. The experimental method well adapted for large scale dynamics study in addition to having the required spatial resolution should on one hand allow averaging among the atoms of the same sort and on the other hand discrimination of different phases of the material, the surface dynamics of which is under investigation.

Using the SPEM we tackled a model system, electromigration of Ga confined film on a GaN (0001) surface aiming at shedding light on the following unanswered questions: (i) how the surface microstructure may affect the lateral distribution of the electromigrating species and (ii) how the superimposed thermal diffusion alters this distribution. Analysis of Ga 3d lineshapes allows identification of the Ga states on the GaN surface, the first ML or interface gallium, called Ga-I phase and the metallic one, and Ga-M phase which grows on top. This has enabled us to follow spatial distribution of the two states inside the patch during and after thermal treatments and electromigration.

The experimental results evidence that below $400{ }^{\circ} \mathrm{C}$ the only migrating species are the Ga atoms from the Ga$\mathrm{M}$ phase. The effects of directional electromigration and 'random' thermal diffusion were determined separately. The main temperature effect is the rearrangement of the top metallic Ga layer due to thermally activated diffusion favoring island formation, in a 'homogenous manner', i.e. preserving the concentration profile shape of Ga-M, illustrated in Fig.3 (a), but with decreased intensity.

At temperature below $400{ }^{\circ} \mathrm{C}$, the application of an electric field of $100 \mathrm{~V} / \mathrm{cm}$ across the patch affects only the shape of the Ga-M concentration profile, as shown in Fig. 3(b). In this rearrangement the migration of the Ga$\mathrm{M}$ atoms is confined on top of the 'immobile' interfacial Ga-I phase. The different concentration profiles across the patch reflect the effect of the local film microstructure, in particular the variations in the density and dimensions of the $\mathrm{Ga}$ islands. Only at the temperatures above 400 ${ }^{\circ} \mathrm{C}$ expansion of the patch can be observed as irregular protrusions along the electric field: the borders become penetrable and a situation where 'unrolling carpet' diffusion takes place. Mobile Ga-M species that overcome the Ga-I plateau in the areas having locally small diffusion barrier provide a further way of migration through the decrease of diffusion barrier, and lead to the formation of the protrusions observed in the image in Fig. 3 (c).

\section{Reaction-induced spatial redistribution of $\mathbf{K}$ and Au adlayers deposited on a $\operatorname{Rh}(110)$ surface}

It is well known that the presence of a small amount of a second metal alters the physical and chemical properties of the surface, which has been demonstrated by means of adsorption and reaction studies employing surface sensitive techniques. Along with modification of the surface properties a distinct feature of the metal adatoms is the dependence of their mobility and bonding on the presence of coadsorbates and on the actual geometric surface structure, which in the case of propagating reaction fronts can lead to lateral re-distribution of the modifiers. Crucial for the experimental demonstration of these poorly documented evens are the chemical sensitivity of the XPEEM and SPEM. The advantage of XPEEM-LEEM-LEED to follow in-situ the dynamic processes and to reveal also the complex changes in the local substrate structure was successfully combined with the better spectral resolution of SPEM for detailed characterization of the local chemical state of the substrate and the adsorbed species.

The first observation of reaction front induced transport of a surface modifier was made for $\mathrm{K}$. This is a specific case of adatoms, which are very mobile on metal surfaces and exert very high chemical activity to so-called elec- 
(a)

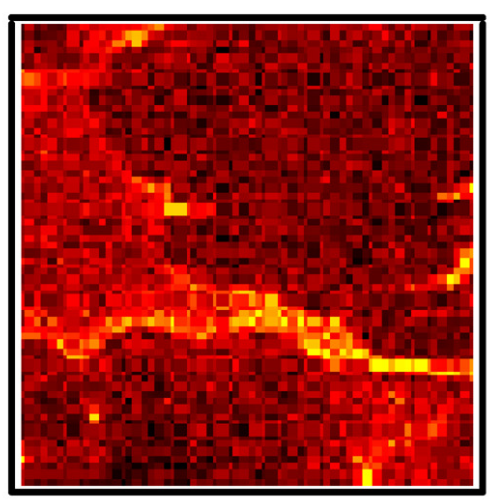

(b)
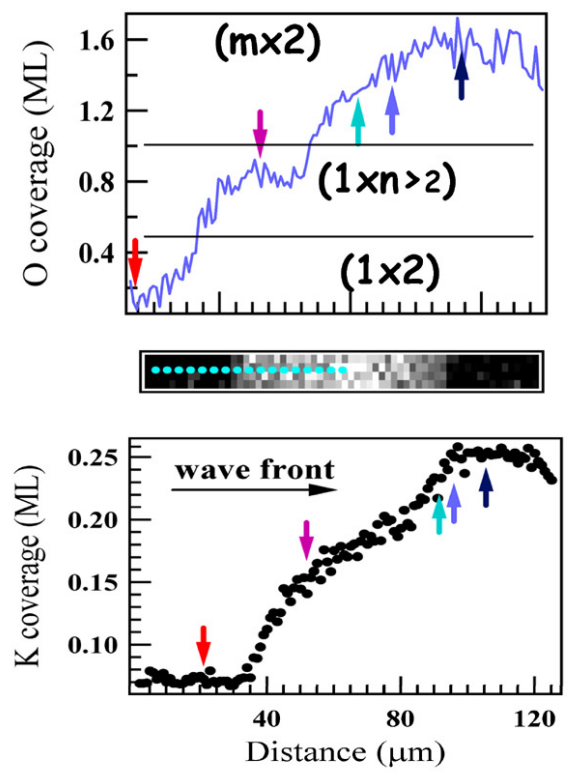

(c)

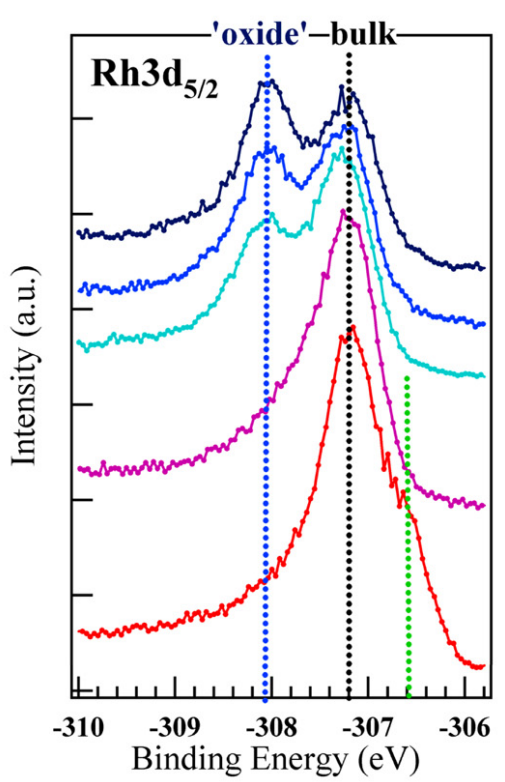

FIG. 4: (a) K 2p SPEM image $\left(64 \times 64 \mu \mathrm{m}^{2}\right)$ illustrating the inhomogeneous distribution of $\mathrm{K}$ induced by the propagating reaction fronts (b) $\mathrm{K}$ and $\mathrm{O}$ concentration profiled measured along the line indicated in the corresponding $\mathrm{K} 2 \mathrm{p}$ map. The local structures measured by imaging microscope in LEEM-LEED mode in the different regions are indicated inside the panel with the $\mathrm{O}$ concentration profile. (c) $\mathrm{Rh} 3 \mathrm{~d}_{5 / 2}$ spectra measured in the spots indicated in the profile plots shown in (b). The two lines indicate the Rh bulk component and the chemically shifted surface component due to coordination of the Rh surface atoms to increasing number of $\mathrm{O}$ adatoms. Reaction conditions: $580 \mathrm{~K}, \mathrm{P}_{\mathrm{O}_{2}}$ and $\mathrm{P}_{H_{2}} \sim 1.5 \times 10^{-7}$ mbar.

tronegative adatoms such as $\mathrm{O}, \mathrm{N}, \mathrm{NO}, \mathrm{CO}$, etc.

Figure 4 illustrates the stationary concentration pattern of $\mathrm{K}$ mapped using SPEM, developed as a result of $\mathrm{K}$ mass transport by the reduction fronts during $\mathrm{H}_{2}+\mathrm{O}_{2}$ reaction on a $\mathrm{Rh}(110)$ surface modified by a submonolayer of $\mathrm{K}$. Within certain reaction conditions (temperature and $\mathrm{O}_{2}$ and $\mathrm{H}_{2}$ gas pressure) these stationary concentration patterns are a stable final state in which potassium is condensed in islands coadsorbed with oxygen. Evidence that the reduction fronts are 'transporting' potassium towards still oxygen-covered areas was the strong enrichment of the forefront with K, easily monitored with SPEM. The stationary patterns can be dissolved by changing the gas environment, e.g. by creation of a reduction ambient, when oxygen is reacting-off, or oxidizing ambient when the entire surface becomes covered with oxygen. The local structure of the substrate inside the condensed $\mathrm{K}+\mathrm{O}$ islands and in the depleted reduced areas outside the islands measured by LEEM-LEED is indicated in the panel illustrating the spatial variation in the $\mathrm{O}$ concentration profile across the condensed island. The chemical state of the substrate is best manifested by the distinct surface core level shift in $\mathrm{Rh} 3 \mathrm{~d}$ core level spectra, reflecting the different coordination of $\mathrm{Rh}$ with oxygen.

Figure 5 shows the $\mathrm{Au} 4 \mathrm{f}$ maps measured with XPEEM in the latest stage of the same reaction on an $\mathrm{Rh}(110)$ surface modified by $\sim 0.5 \mathrm{ML}$ of $\mathrm{Au}$, which was homogeneously distributed before the start of the reaction. The map clearly manifests $\mathrm{Au}$ condensation patterns, similar to the case of $\mathrm{K}$. However, in this case Au has condensed in the O-free areas, reaching maximum coverage of $\sim 0.9$ ML. The local structure measured by LEED shows an
$(1 \times 2)$ reconstructed $\mathrm{Rh}(110)$ in the Au depleted oxidised areas, where $\mathrm{Au}$ coverage has dropped to $\sim 0.2 \mathrm{ML}$, the streaks being related to the $\mathrm{O}$ disorder, induced by the presence of Au. However in contrast to the case of $\mathrm{K}$ condensation XPEEM and LEEM measurements showed that the patterned $\mathrm{Au} / \mathrm{Rh}$ interface is preserved in oxygen ambient and is dissolved quickly only under reduction conditions restoring the initial homogeneous distribution of Au. The advantage of using the combination XPEEM-LEEM-LEED for these dynamic systems is that once proved the presence of chemical contrast and correlated with the LEEM images, we were able to study in details the ignition and propagation of the reaction front and the response of the local surface structure utilizing the LEEM imaging combined with LEED, which provides additional structural information with resolution better than that achieved in XPEEM mode. For example, LEED has revealed that the heterogeneity in the Au coverage leads to different local reactivity so that exposed to oxidizing atmosphere the depleted $\mathrm{Au}$ areas undergo the $(1 \times 4)-(1 \times 5)$ reconstructions, whereas the enriched with $\mathrm{Au}$ areas $(1 \times 2)$. Detailed spectroscopic measurements with SPEM have revealed quite complex response of the $\mathrm{Rh} 3 \mathrm{~d}$ core levels to the local oxygen and Au coverage, indicating that the strongest $\mathrm{Au}-\mathrm{Rh}$ interactions occur at low $\mathrm{Au}$ coverages, i.e. below $0.25 \mathrm{ML}$.

The mechanism of the $\mathrm{K}$ and $\mathrm{Au}$ condensation processes can been best described considering the following key factors:

( 1 ) Potential energy surface of the adsorbed Au and K on O-covered and O-free $\mathrm{Rh}$ surface. For the case 


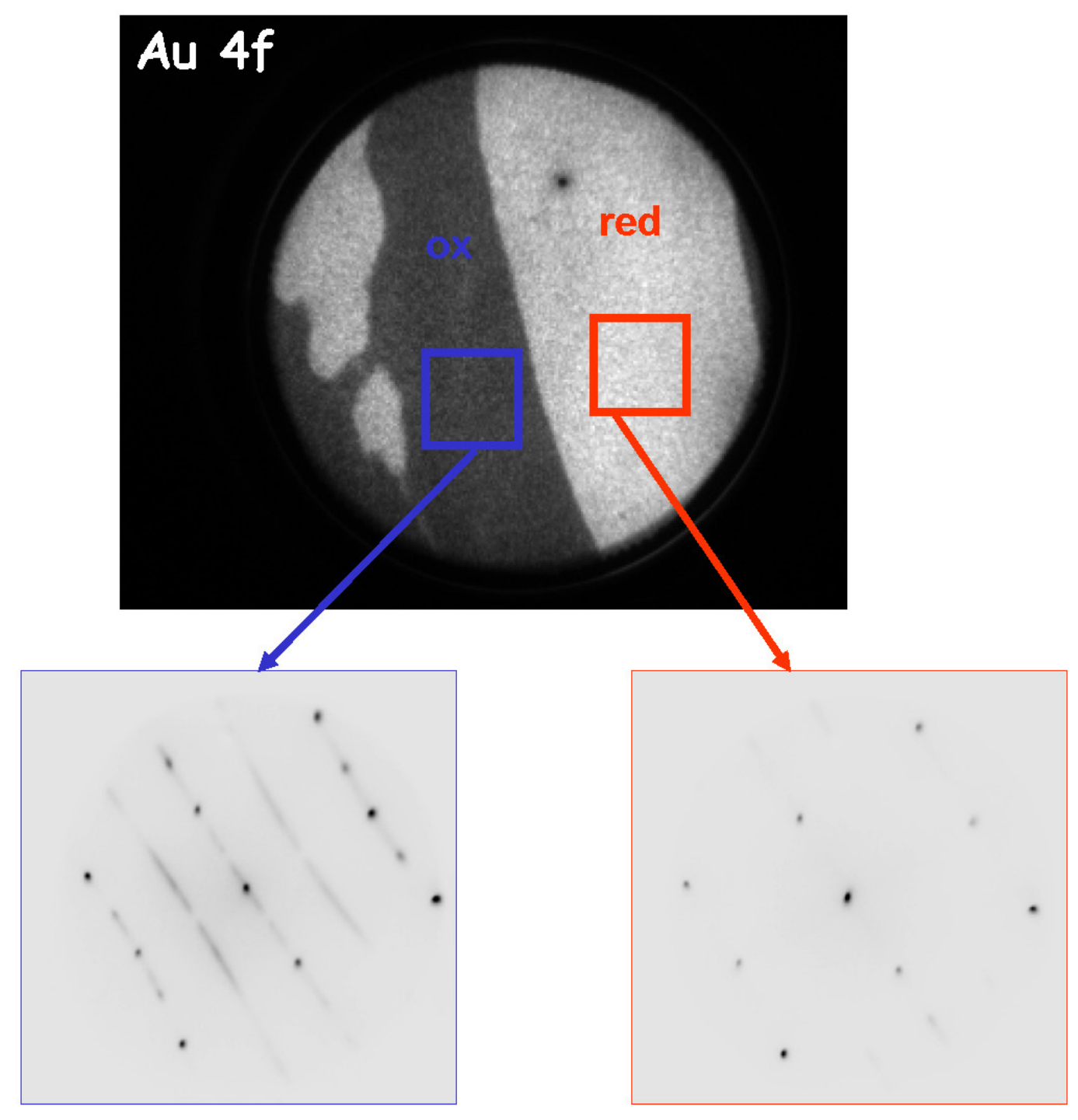

FIG. 5: $\mathrm{Au}$ 4f XPEEM image $(\mathrm{FOV}=10 \mu \mathrm{m})$ illustrating the accumulation of $\mathrm{Au}$ in the reduced areas. The LEED of reduced Au-rich and oxidized Au-depleted areas show the corresponding lateral heterogeneity of the Rh surface structure. Reaction conditions: $550 \mathrm{~K}, \mathrm{P}_{\mathrm{O}_{2}}$ and $\mathrm{P}_{\mathrm{H}_{2}} \sim 3 \times 10^{-7}$ mbar.

of $\mathrm{K}$ the O-covered surface is energetically favored, whereas in the case of $\mathrm{Au}$ it is the reduced one.

( 2 ) Mobility of the Au and $\mathrm{K}$ on an O-covered and Ofree Rh surface. For both adatoms it is lower in the presence of oxygen, i.e. $\mathrm{K}$ and $\mathrm{Au}$ diffuse more rapidly on the reduced surface.

( 3 ) Local reactivity of the condensed phases. The reactivity of oxygen in the condensed $\mathrm{K}+\mathrm{O}$ phase towards hydrogen is strongly reduced. The chemically 'inert' Au exerts blocking effect and reduces the oxygen adsorption rate.

\section{CONCLUSIVE REMARKS AND OUTLOOK}

The selected results present only some of the important fields where the synchrotron radiation photoemission microscopy has provided valuable information. Some of the near-future upgrades for improving the performance of scanning microscopes are increasing the lateral resolution using better optical elements, adapting sample cooling systems in order to probe phenomena dependent on the sample temperature, developing of faster detection systems.

\section{Acknowledgments}

The results of which are used for illustration of microscopy activity at ELETTRA are obtained with the valuable contribution of $\mathrm{A}$. Barinov, A. Böttcher, H. Conrad, L. Gregoratti, S. Günther, R. Imbihl, H. Marbach, A. Locatelli and S. Heun who initiated and actively participated in the different projects. This work was supported by Sincrotrone Trieste SCpA and partially by the EEC contract HPRI-CT-1999-00033. 
[1] S. Gunther, B. Kaulich, L. Gregoratti and M. Kiskinova, Prog. Surf. Sci. 70, 1 (2002), and references therein.

[2] E. Bauer, Rep. Prog. Phys. 57, 895 (1994).

[3] H. Over, Y.D. Kim, A.P. Seitsonen, Science 287, 1474 (2000).

[4] A. Böttcher, H. Conrad and H. Niehus, Surf. Sci. 452, 125 (2000).

[5] A. Böttcher ,U. Starke, H. Conrad, R. Blume, H. Niehus, L. Gregoratti, B. Kaulich, A. Barinov, M. Kiskinova, J. Chem. Phys. 117, 8104 (2002).

[6] A. Barinov, L. Gregoratti, B. Kaulich, M. Kiskinova,
ChemPhysChem, 3, 1019 (2002), and references therein. [7] S. Günther, H. Marbach, R. Hoyer. et. al., J. Electr. Spectr. Rel. Phenom. 114, 989 (2001).

[8] S. Günther, H. Marbach, R. Hoyek, R. Imbihl , L. Gregoratti, M. Kiskinova, Surf. Rev. Lett. 9, 751 (2002).

[9] S. Günther, H. Marbach, R. Hoyek, R. Imbihl , L. Gregoratti, M. Kiskinova, J. Chem. Phys. 117, 2923 (2002).

[10] A. Locatelli, S. Heun, M. Kiskinova, Surf. Sci. in press and in preparation. 